\title{
RECUPERAÇÃO DE BIXINA DAS SEMENTES DE URUCUM (Bixa orellana L.) UTILIZANDO UMA NOVA CONFIGURAÇÃO PARA O LEITO DE JORRO
}

\author{
T. TAHAM ${ }^{1 *}$, D. O. SILVA ${ }^{1}$, M. A. S. BARROZO ${ }^{1}$ \\ ${ }^{1}$ Universidade Federal de Uberlândia, Faculdade de Engenharia Química \\ e-mail: thiago.taham@iftm.edu.br
}

\begin{abstract}
RESUMO
A bixina é o carotenoide mais utilizado na indústria alimentícia. É tradicionalmente extraída das sementes de urucum por meio de soluções alcalinas, fricção em óleo vegetal aquecido ou por meio de solventes orgânicos. Estes métodos, além de exigir uma operação unitária subsequente de separação da bixina, podem gerar resíduos tóxicos. Assim, a extração mecânica torna-se um meio atrativo para a obtenção de pó rico em bixina a partir das sementes de urucum, sendo caracterizado como um método "verde". Este trabalho objetivou investigar a extração mecânica da bixina das sementes de urucum utilizando uma unidade de leito de jorro com um anteparo de altura regulável. Foi realizado um estudo experimental para avaliar a influência de três variáveis (carga de sementes, altura do anteparo e a vazão de ar) nas respostas (massas de pó e bixina extraídos e o percentual de bixina no pó). Os efeitos foram calculados utilizando regressão múltipla. As melhores condições de extração foram determinadas utilizando um algoritmo de evolução diferencial. Foram identificadas as condições que levaram às melhores eficiências de extração (para o pó e a bixina) e à maior pureza de pó obtido (acima de 65\%). Os valores experimentais obtidos apresentaram baixos desvios em relação aos correspondentes preditos pelo modelo.
\end{abstract}

\section{INTRODUÇÃO}

O urucum (Bixa orellana L.) é um arbusto característico da floresta amazônica de várzea, cujas sementes produzem um pigmento avermelhado, dependendo de sua concentração em solução (NOBRE et al., 2006). Nativo da parte tropical sul-americana, seus frutos são usados como ingredientes alimentícios há séculos (CHUYEN et al., 2012). Este corante existe nas formas hidrossolúvel e lipossolúvel, podendo ser empregado em grande número de produtos alimentícios. Seu baixo custo de produção e baixa toxicidade fazem dele um pigmento muito atrativo e conveniente, em substituição a corantes sintéticos (AGNER et al., 2004). O corante de urucum é bastante utilizado na indústria alimentícia, mais particularmente em lácteos e carnes. O êxito de uso no setor é devido à comparativa instabilidade dos corantes sintéticos nestas aplicações. Comercialmente três métodos de extração da bixina do urucum são mais utilizados: a extração alcalina, a extração em óleo, que resulta na remoção da bixina e de outros materiais coloridos, e a extração com solvente, que resulta na forma mais pura do pigmento bixina (PRENTICEHERNANDEZ; RUSIG, 1992). As extrações com solvente orgânico ou solução alcalina geram resíduos causadores de problemas ambientais, além de requererem uma operação unitária subsequente para remover o solvente ou solução utilizada na extração, elevando o custo total de energia (ALBUQUERQUE; 
MEIRELES, 2012). A extração em óleo vegetal aquecido produz suspensões mais concentradas de pigmentos, mas que podem conter produtos de degradação, haja vista que a extração é realizada em temperaturas maiores que $100^{\circ} \mathrm{C}$ (MCKEOWN; MARK, 1962).

Várias técnicas vêm sendo estudadas para desenvolver tecnologias limpas de extração, com menores impactos ambientais. $\mathrm{Na}$ extração da bixina das sementes de urucum podem ser destacados estudos utilizando $\mathrm{CO}_{2}$ supercrítico (PRENTICEHERNANDEZ; RUSIG, 1992; CHAO et al., 1991; SILVA et al., 2008; ANDERSON et al., 1997; TAHAM; CABRAL; BARROZO, 2015), extração utilizando sementes desengorduradas (ALBUQUERQUE; MEIRELES, 2012; RODRIGUES et al., 2014; TAHAM; CABRAL; BARROZO, 2015), extração assistida por ultrassom (ALBUQUERQUE; MEIRELES, 2012; RODRIGUES et al., 2014; NAVES; ZABOT; MEIRELES, 2015) e melhorias na extração convencional, utilizando água ou etanol como solventes (SHUSHAMA et al., 2003; SATYANARAYANA; PRABHAKARA; RAO, 2003; RODRIGUES et al., 2014; TAHAM; CABRAL; BARROZO, 2015). Um método físico alternativo é a fricção mecânica das sementes para remover a camada superficial onde o pigmento se encontra. Este tipo de extração mecânica traz algumas vantagens, como a redução do tempo de extração, eliminação (completa ou parcial) do uso de solventes, aumento no rendimento e melhoria na qualidade dos extratos (CUNHA et al., 2009; BARROZO; SANTOS; CUNHA, 2013). Esta tecnologia envolve o uso de equipamentos relativamente simples, como um moinho de bolas ou um leito de jorro que, além de serem de fácil instalação, podem ser utilizados em locais próximos às plantações, agregando valor ao produto a ser comercializado. O leito de jorro ainda se destaca por permitir um maior controle do processo (BARROZO; SANTOS; CUNHA, 2013).

Alguns trabalhos já foram desenvolvidos com o objetivo de extrair mecanicamente a bixina das sementes de urucum utilizando o leito de jorro. Passos et al. (1998) estudaram a taxa de produção de pó a partir das sementes do urucum utilizando uma unidade cônica de leito de jorro. Cunha et al. (2009) concluíram que a presença de um tubo draft no interior do leito foi a variável que mais afetou a extração de pó. Barrozo; Santos; Cunha (2013) quantificaram aspectos fluidodinâmicos e as massas extraídas de pó e bixina em uma unidade cônico-cilíndrica. Em todos os estudos conduzidos ficou claro que a vazão de ar e a presença do tubo draft foram os parâmetros de processo que mais fortemente influenciaram a recuperação de bixina, uma vez que aumentam o atrito entre as sementes.

Objetivando aumentar o rendimento de extração da bixina a partir das sementes de urucum, o presente estudo propôs uma nova configuração para uma um leito de jorro utilizando um tubo draft: o leito de jorro com anteparo de altura regulável. A influência da posição do anteparo, composto por uma tela de aço inoxidável, foi investigada, juntamente com a massa inicial das sementes e a vazão de ar. As melhores configurações destas variáveis foram determinadas por meio de planejamento experimental e análise de regressão, bem como por meio de otimização de processos utilizando a técnica de algoritmos genéticos.

\section{MATERIAIS E MÉTODOS}

\subsection{Caracterização da Matéria-Prima}

As sementes de urucum foram compradas em comércio local da cidade de Rio Vermelho/MG e estocadas a $-18^{\circ} \mathrm{C}$ e protegidas da luz, em sacos escuros de polietileno, até que as análises de qualidade fossem feitas. 
A umidade, teor de proteínas, cinzas e lipídios foram determinados segundo métodos Oficiais AOAC (1997). O teor de carboidratos foi determinado por diferença. O conteúdo de bixina foi determinado por meio de extração convencional com solvente, que consiste na repetida extração do corante das sementes com clorofórmio a $50^{\circ} \mathrm{C}$, até o completo esgotamento das mesmas. Estas condições foram baseadas no trabalho de Silva; Cavalcanti; Sobral (1994) cujos resultados mostraram que, além do bom desempenho do clorofórmio como solvente, temperaturas abaixo de $80^{\circ} \mathrm{C}$ causam mínima degradação na bixina. $O$ teor de bixina foi, então, determinado através de método espectrofotométrico recomendado pela FAO/WHO (2004). A absorbância das amostras foi medida em cubeta de quartzo de $1 \mathrm{~cm}$ a $487 \mathrm{~nm}$, utilizando um espectrofotômetro UV-visível. Todas as medidas foram executadas em triplicata. A porcentagem de bixina foi calculada, então, de acordo com a lei de Lambert-Beer, utilizando o coeficiente de absortividade para a bixina $\sum_{1 \mathrm{~cm}}^{1 \%}={ }_{3090 \text {. }}$

\subsection{Extração em Leito de Jorro}

As extrações em leito de jorro foram realizadas em uma unidade experimental apresentada, esquematicamente, na Figura 1:

Figura 1 - O leito de jorro

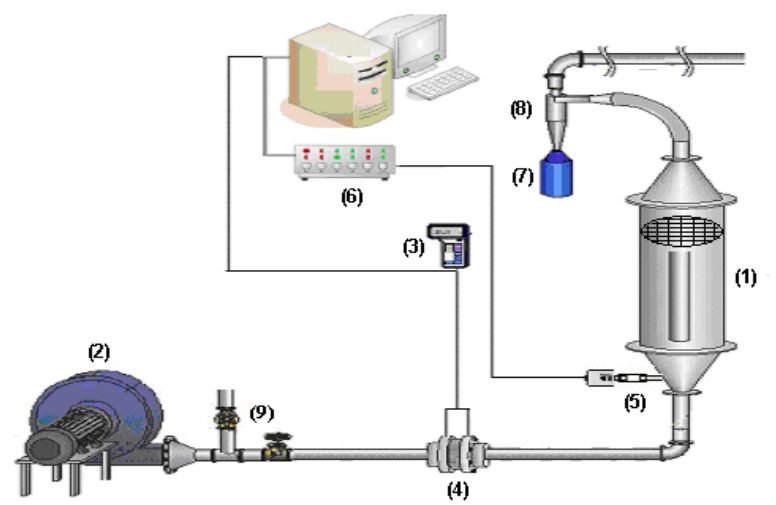

Fonte: Cunha et al. (2009)
A Figura 1 mostra o leito de jorro, composto por uma estrutura de base cônica e estrutura cilíndrica (1) com visor em vidro ao longo do corpo cilíndrico, um compressor de ar de 7,5 hp (2), uma placa de orifício (4) ligada a um transdutor de pressão (3). O pó de bixina extraído foi coletado em frasco âmbar (7), protegido da luz, conectado ao underflow de um ciclone, tipo Stainmard de $10 \mathrm{~cm}$ de diâmetro (8). Os sinais do transdutor de pressão foram transmitidos a um computador por meio de uma placa de aquisição de dados (6) e processados utilizando o software LabVIEWTM 7.1. As dimensões do leito de jorro são: ângulo da base cônica de $60^{\circ}$, orifício de entrada do ar de $3,5 \mathrm{~cm}$, diâmetro da seção cilíndrica de $21 \mathrm{~cm}$ e comprimento igual a $70 \mathrm{~cm}$. A este aparato foi acrescentada um tubo draft cilíndrico, feito com uma tela de aço inoxidável $8 \mathrm{MESH}$ (dimensões 4,5 $\mathrm{cm}$ de diâmetro, $10 \mathrm{~cm}$ de comprimento, posicionado a $4 \mathrm{~cm}$ da entrada de ar). A presença do tubo draft, além de reduzir a vazão de ar necessária para o estabelecimento do jorro, aumenta o fator de atrito e incrementa a extração. A distância de $4 \mathrm{~cm}$ foi escolhida baseada no resultado obtido no trabalho experimental de Barrozo, Cunha e Santos (2013). Uma modificação feita no equipamento foi a inclusão de um anteparo no interior da parte cilíndrica, composto por uma tela circular, de aço inoxidável $8 \mathrm{MESH}$, com altura regulável. Esta tela permitia a passagem do pó, mas não das sementes, e contribuiu, também, para aumentar o atrito e melhorar o rendimento de extração.

Testes preliminares foram conduzidos para determinar as mínimas condições de jorro $\left(\mathrm{Q}_{\mathrm{jm}}\right)$ para cada carga de sementes. $\mathrm{O}$ jorro mínimo é determinado reduzindo a vazão de ar após o estabelecimento do regime de jorro; o ponto em que há colapso da fonte.

Um planejamento experimental foi realizado para analisar o efeito de três variáveis independentes em três respostas. As três variáveis independentes escolhidas foram 
a massa de sementes $\left(\mathrm{M}_{\mathrm{s}}\right)$, a posição do anteparo $\left(\mathrm{H}_{\mathrm{a}}\right)$, e a relação entre a vazão de ar e a vazão de jorro mínimo $\left(\mathrm{Q} / \mathrm{Q}_{\mathrm{jm}}\right)$. As três respostas foram a massa de pó $\left(\mathrm{M}_{\mathrm{p}}\right)$, a massa de bixina $\left(\mathrm{M}_{\mathrm{b}}\right)$ e a pureza do pó, representada pela porcentagem de bixina $\left(\%_{\text {bix }}\right)$. Também foram conduzidas três replicas no ponto central e seis pontos axiais, totalizando 17 ensaios, como mostra a Tabela 1.

Tabela 1 - Planejamento Experimental

\begin{tabular}{cccc}
\hline $\mathbf{E x p}$ & $\mathbf{M}_{\mathbf{s}}(\mathbf{g})$ & $\mathbf{H}_{\mathbf{a}}(\mathbf{c m})$ & $\mathbf{Q} / \mathbf{Q}_{\mathbf{j m}}$ \\
\hline $\mathbf{1}$ & 1700 & 30 & 1,24 \\
$\mathbf{2}$ & 1700 & 30 & 1,80 \\
$\mathbf{3}$ & 1700 & 60 & 1,24 \\
$\mathbf{4}$ & 1700 & 60 & 1,80 \\
$\mathbf{5}$ & 2300 & 30 & 1,24 \\
$\mathbf{6}$ & 2300 & 30 & 1,80 \\
$\mathbf{7}$ & 2300 & 60 & 1,24 \\
$\mathbf{8}$ & 2300 & 60 & 1,80 \\
$\mathbf{9}$ & 1500 & 45 & 1,525 \\
$\mathbf{1 0}$ & 2500 & 45 & 1,525 \\
$\mathbf{1 1}$ & 2000 & 20 & 1,525 \\
$\mathbf{1 2}$ & 2000 & 70 & 1,525 \\
$\mathbf{1 3}$ & 2000 & 45 & 1,05 \\
$\mathbf{1 4}$ & 2000 & 45 & 2,00 \\
$\mathbf{1 5}$ & 2000 & 45 & 1,525 \\
$\mathbf{1 6}$ & 2000 & 45 & 1,525 \\
$\mathbf{1 7}$ & 2000 & 45 & 1,525 \\
\hline
\end{tabular}

Os experimentos foram conduzidos seguindo uma ordem aleatória. Amostras foram recolhidas ao longo do tempo, para obter-se a cinética de extração, que durou 5 horas cada. Após esse tempo, pequenos fragmentos de casca começavam a ser extraídos, reduzindo a qualidade dos extratos. Cada amostra recolhida foi estocada a $-18{ }^{\circ} \mathrm{C}$ e protegida da luz, até futuras análises.

\subsection{Otimização da Extração usando ED}

Equações para representar as respostas analisadas (massa de pó, massa de bixina e pureza do pó) como funções das variáveis independentes foram obtidas por meio do tratamento das respostas experimentais. A otimização do modelo proposto pela análise estatística foi feita utilizando o método de Evolução Diferencial (DE), baseado em operações vetoriais que geram potenciais candidatos à resolução dos problemas de otimização (PRICE; STORN, 1997). Dois novos experimentos foram conduzidos para verificar, fisicamente, o quanto a resposta experimental, nas condições otimizadas, se aproximava da estimativa do modelo.

\section{RESULTADOS E DISCUSSÕES}

\subsection{Caracterização das sementes}

A umidade média das sementes foi de $8,7 \%$ (base úmida), adequada para armazenamento. O conteúdo de cinzas, proteínas, óleo e carboidratos foram respectivamente, $4,32 \%$; $11,34 \% ; 5,40 \%$ e $70,24 \%$. Os valores obtidos estão similares a outros trabalhos encontrados na literatura (SILVA et al., 1994; ALBUQUERQUE; MEIRELES, 2012). A extração convencional utilizando clorofórmio como solvente resultou em um conteúdo de bixina nas sementes igual a 4,5\%.

\subsection{Extração Utilizando Leito de Jorro}

A primeira etapa da execução dos experimentos foi a determinação experimental do jorro mínimo para cada carga de sementes. Os resultados estão apresentados na Tabela 2.

Tabela 2 - Vazões de Jorro mínimo

\begin{tabular}{cc}
\hline $\mathbf{M}_{\mathbf{s}}(\mathbf{g})$ & $\mathbf{Q}_{\mathbf{j m}}\left(\mathbf{m}^{\mathbf{3}} \mathbf{h}\right)$ \\
\hline 1500 & 46 \\
1700 & 48 \\
2000 & 51 \\
2300 & 54 \\
2500 & 56 \\
\hline
\end{tabular}

Os valores encontrados foram utilizados para determinar a vazão de ar em cada experimento. Os experimentos foram, então, conduzidos de acordo com o planejamento experimental delineado a fim de se estudar o processo dentro das faixas selecionadas. Os 
resultados do planejamento obtidos para as três respostas estão mostrados na Tabela 3:

Tabela 3 - Respostas Experimentais

\begin{tabular}{ccccc}
\hline $\mathbf{E x p}$ & $\mathbf{H}_{\mathbf{f}} / \mathbf{H}_{\mathbf{a}}$ & $\mathbf{M}_{\mathbf{p}}(\mathbf{g})$ & $\mathbf{M}_{\mathbf{b}}(\mathbf{g})$ & $\mathbf{\%}_{\mathbf{b i x}}$ \\
\hline $\mathbf{1}$ & 1,00 & 21,65 & 8,08 & 37,30 \\
$\mathbf{2}$ & 2,33 & 66,67 & 19,84 & 29,75 \\
$\mathbf{3}$ & 0,50 & 20,02 & 10,45 & 52,21 \\
$\mathbf{4}$ & 1,17 & 53,13 & 17,12 & 32,23 \\
$\mathbf{5}$ & 1,00 & 28,09 & 11,72 & 41,73 \\
$\mathbf{6}$ & 2,17 & 89,24 & 27,67 & 31,01 \\
$\mathbf{7}$ & 0,50 & 27,74 & 13,52 & 48,73 \\
$\mathbf{8}$ & 1,33 & 97,63 & 23,17 & 23,74 \\
$\mathbf{9}$ & 1,22 & 25,82 & 11,00 & 42,60 \\
$\mathbf{1 0}$ & 1,22 & 74,50 & 24,12 & 32,38 \\
$\mathbf{1 1}$ & 2,50 & 36,60 & 12,32 & 33,67 \\
$\mathbf{1 2}$ & 0,71 & 40,19 & 11,49 & 28,59 \\
$\mathbf{1 3}$ & 0,56 & 13,13 & 7,85 & 59,80 \\
$\mathbf{1 4}$ & 1,56 & 96,25 & 28,91 & 30,04 \\
$\mathbf{1 5}$ & 1,11 & 41,57 & 14,30 & 34,40 \\
$\mathbf{1 6}$ & 1,11 & 40,24 & 13,39 & 33,28 \\
$\mathbf{1 7}$ & 1,11 & 39,48 & 14,10 & 35,72 \\
\hline
\end{tabular}

Para melhor visualização da influência da altura do anteparo $\left(\mathrm{H}_{\mathrm{a}}\right)$ nas respostas, foi calculada a relação entre a máxima altura atingida pelas sementes em cada experimento (altura da fonte $-\mathrm{H}_{\mathrm{f}}$ ) e a altura do anteparo $\left(\mathrm{H}_{\mathrm{f}} / \mathrm{H}_{\mathrm{a}}\right)$. A altura da fonte foi aferida por meio de observação experimental através do visor na parte cilíndrica, sem a presença do anteparo. Altos valores da relação $\mathrm{H}_{\mathrm{f}} / \mathrm{H}_{\mathrm{a}}$ mostram a sementes se chocando mais fortemente no anteparo, valores menores que 1 mostram que as sementes não o tocam, anulando seu efeito. Os valores da relação $\mathrm{H}_{\mathrm{f}} / \mathrm{H}_{\mathrm{a}}$ estão apresentados na Tabela 3.

Pode ser observado que os maiores valores obtidos para as massas de pó e bixina foram obtidos nos experimentos 6, 8, e 14, nos quais ou está presente uma alta vazão. Este fato indica que a vazão de ar tem uma influência maior sobre as respostas do que a massa das sementes. Nos 3 ensaios é possível observar, também, que a relação $\mathrm{H}_{\mathrm{f}} / \mathrm{H}_{\mathrm{a}}$ é maior do que 1 , ou seja, o fato de as sementes terem alcançado a tela foi significativo. Os menores valores, por sua vez, foram obtidos nos experimentos 1, 3 e 13. Estes três testes tiveram em comum a combinação entre baixas vazões de ar e cargas de semente, comportamento que está em pleno acordo com os resultados encontrados por Barrozo; Santos; Cunha (2013).

As mais altas porcentagens de bixina no pó obtido foram obtidas nos experimentos 3, 7 e 13. Estes experimentos tiveram em comum baixas vazões de ar aliadas a valores da relação $\mathrm{Hf} / \mathrm{H}_{\mathrm{a}}<1$, ou seja, as sementes não atingiram o anteparo. Pode-se concluir, então, que para se obter um pó com alta pureza, deve-se aplicar nas sementes baixas vazões de ar e priorizar o efeito de atrito entre elas, isolando o efeito do anteparo. Pode-se concluir que o aumento do efeito de choque das sementes contra o anteparo $\left(\mathrm{H}_{\mathrm{f}} / \mathrm{H}_{\mathrm{a}}>1\right)$ aumenta tanto o volume de corante extraído como o de outros compostos, reduzindo a pureza. De fato, em alguns experimentos nesta condição, foi possível observar pequenos fragmentos da casca da semente presentes no pó - especialmente nas últimas horas da extração. É importante ressaltar que estes fragmentos são extraídos após a remoção da camada de corante, o que reduz a pureza e o valor comercial do pó obtido. A Figura 2 mostra as sementes dentro do leito, após o experimento 14.

Figura 2 - Sementes após extração

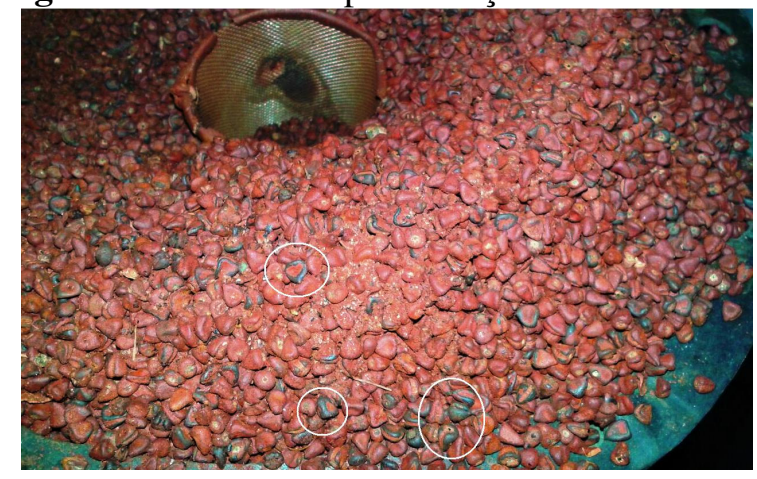

Nesta figura pode-se perceber (circulado em branco) o esgotamento da 
camada de corante das sementes após a extração. Em muitas sementes, nota-se a quase completa remoção do corante. Esta foto foi tirada após a execução do experimento 14, em que foi utilizada a mais severa condição de vazão de ar do planejamento experimental. A esta análise pode-se acrescentar a observação da cinética de extração mostrada na Figura 3, que ajuda a corroborar a hipótese do quase completo esgotamento da camada de corante das sementes de urucum:

Figura 3 - Cinética de extração da bixina

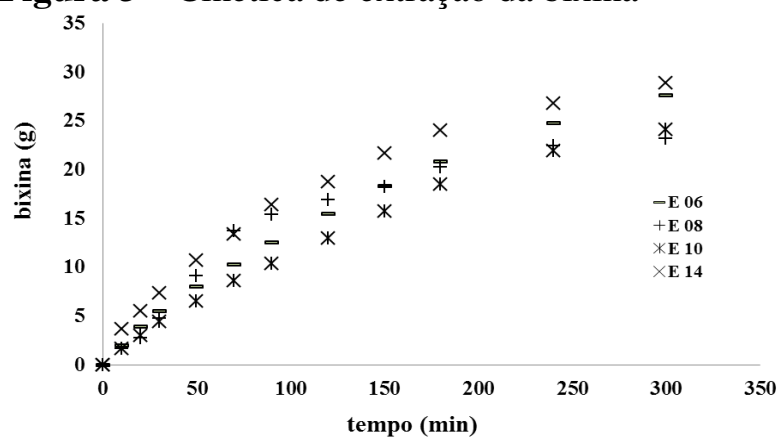

Os quatro experimentos apresentados no gráfico $(6,8,10$ e 14) foram escolhidos por terem apresentado os mais altos rendimentos de bixina, de acordo com a Tabela 3. No gráfico pode ser observada uma extração mais acentuada no início, seguida por uma mudança na inclinação da sequência de pontos, indicando uma redução de valor comercial na taxa de extração a partir de 300 minutos.

No presente estudo, a análise estatística foi feita por regressão múltipla, objetivando obter correlações empíricas dos dados experimentais capazes de predizer as respostas selecionadas - massa de pó $\left(\mathrm{M}_{\mathrm{p}}\right)$, massa de bixina $\left(\mathrm{M}_{\mathrm{b}}\right)$ e porcentagem de bixina no pó $\left(\%_{\text {bix }}\right)$ - como funções das variáveis independentes na sua forma codificada. As correlações matemáticas obtidas para cada resposta estão dadas, em notação matricial, nas equações (1) - $\mathrm{M}_{\mathrm{p}}$, (2) - $\mathrm{M}_{\mathrm{b}} \mathrm{e}(3)$ - \% $\%_{\text {bix }}$ :

$\mathrm{Mp}=39,55+\mathrm{X}^{\prime} \mathrm{b}_{1}+\mathrm{X}^{\prime} \mathrm{B}_{1} \mathrm{X}$ $b_{1}=\left[\begin{array}{l}12,3 \\ 0,0 \\ 25,0\end{array}\right] ; X=\left[\begin{array}{l}X_{1} \\ X_{2} \\ X_{3}\end{array}\right] ; B_{1}=\left[\begin{array}{ccc}4,1 & 1,1 & 3,6 \\ 1,1 & 0,0 & 0,0 \\ 3,6 & 0,0 & 5,5\end{array}\right]$

$\mathrm{Mb}=13,78+\mathrm{X}^{\prime} \mathrm{b}_{2}+\mathrm{X}^{\prime} \mathrm{B}_{2} \mathrm{X}$

$b_{2}=\left[\begin{array}{l}3,1 \\ 0,0 \\ 5,8\end{array}\right] ; X=\left[\begin{array}{l}X_{1} \\ X_{2} \\ X_{3}\end{array}\right] ; B_{2}=\left[\begin{array}{ccc}1,4 & 0,0 & 0,5 \\ 0,0 & -0,6 & -0,7 \\ 0,5 & -0,7 & 1,7\end{array}\right]$

$\%$ bix $=35,83+X^{\prime} b_{3}+X^{\prime} B_{3} X$

$b_{3}=\left[\begin{array}{l}-1,9 \\ 0,0 \\ -8,1\end{array}\right] ; X=\left[\begin{array}{l}X_{1} \\ X_{2} \\ X_{3}\end{array}\right] ; B_{3}=\left[\begin{array}{ccc}0,0 & -1,0 & 0,0 \\ -1,0 & -1,6 & -1,8 \\ 0,0 & -1,8 & 3,2\end{array}\right]$

Nas equações (1)-(3), as variáveis $M_{\mathrm{s}}$ $(\mathrm{g}), \quad \mathrm{H}_{\mathrm{a}}(\mathrm{cm})$ e $\mathrm{Q} / \mathrm{Q}_{\mathrm{jm}}$ (adimensional) receberam a nomenclatura de $\mathrm{X}_{1}, \mathrm{X}_{2}$ e $\mathrm{X}_{3}$, que correspondem às respectivas variáveis codificadas adimensionais, que seguem mostradas nas equações (4)-(6):

$\mathrm{X}_{1}=\frac{M_{s}-2000}{300}$

$\mathrm{X}_{2}=\frac{H_{a}-45}{15}$

$\mathrm{X}_{3}=\frac{\left(Q / Q_{j m}\right)-1,525}{0,28}$

As equações (1)-(3) apresentam apenas os termos estatisticamente significativos, a $10 \%$ de significância; os termos não significativos foram substituídos por zero nas equações. $\mathrm{O}$ coeficiente de determinação $\left(\mathrm{R}^{2}\right)$ das três análises foram $99,1 \%$ para a Eq. 1 $\mathrm{M}_{\mathrm{p}}, 98,4 \%$ para a Eq. $2-\mathrm{M}_{\mathrm{b}}$ e $93,8 \%$ para a Eq. 3 - \% bix. As análises residuais dessas regressões mostraram que os resíduos estavam distribuídos de forma independente 
de acordo com uma distribuição normal com média zero e desvio fixo.

A análise estatística mostrou que a vazão de ar tem a mais forte influência (tanto linear quanto quadrática) nas respostas "massa de pó" e "massa de bixina", seguida da influência da massa inicial das sementes. A combinação entre uma baixa altura do anteparo com altas cargas de semente aumentou a recuperação de bixina. Altura elevada do anteparo combinada com baixas vazões, por sua vez, retornou as maiores purezas de pós dentre as condições testadas.

\subsection{Otimização da Extração usando ED}

Objetivando otimizar o processo de extração considerando as respostas analisadas, duas situações foram destacadas:

Caso 1: o objetivo principal aqui foi maximizar a produção de bixina, que apenas pode ser atingido maximizando, também, a produção de pó. Uma restrição de $30 \%$ de conteúdo de bixina no pó foi imposta ao algoritmo de modo a garantir que o produto final mantivesse seu valor comercial.

Caso 2: neste caso o objetivo principal foi, por sua vez, produzir um pó com a máxima pureza, maximizando a porcentagem de bixina no pó independente da quantidade produzida.

Os parâmetros usados pelo algoritmo genético estão plenamente detalhados no trabalho de Silva et al. (2012). Cada resultado de otimização foi gerado 10 vezes, a fim de se obter uma média.

A Tabela 4 apresenta os resultados de otimização dados pela análise do modelo matemático, com os valores reais das variáveis em suas condições otimizadas (de acordo com as equações 1 a 3 ):

Tabela 4. Níveis e resultados preditos por ED

\begin{tabular}{cccc}
\hline $\begin{array}{c}\text { Parâmetros } \\
\text { otimizados }\end{array}$ & $\mathbf{M}_{\mathbf{s}}(\mathbf{g})$ & $\mathbf{H}_{\mathbf{a}}(\mathbf{c m})$ & $\mathbf{Q} / \mathbf{Q}_{\mathbf{j m}}$ \\
\hline Caso 1 & 2500 & 42 & 2,0 \\
Caso 2 & 1500 & 70 & 1,05 \\
\hline
\end{tabular}

A fim de validar experimentalmente as condições dadas pelo algoritmo, dois novos experimentos foram conduzidos seguindo os parâmetros operacionais otimizados; os resultados preditos e experimentais estão apresentados na Tabela 5.

Tabela 5. Resultados Preditos e Experimentais em configurações otimizadas

\begin{tabular}{ccccccc}
\hline & \multicolumn{2}{c}{$\mathrm{M}_{\mathrm{p}}(\mathrm{g})$} & \multicolumn{2}{c}{$\mathrm{M}_{\mathrm{b}}(\mathrm{g})$} & \multicolumn{2}{c}{$\%_{\text {bix }}$} \\
\cline { 2 - 7 } & $\mathrm{Y}_{\text {pred }}$ & $\mathrm{Y}_{\exp }$ & $\mathrm{Y}_{\text {pred }}$ & $\mathrm{Y}_{\exp }$ & $\mathrm{Y}_{\text {pred }}$ & $\mathrm{Y}_{\exp }$ \\
\hline 1 & 141,8 & 134,9 & 42,5 & 47,5 & 30,0 & 35,2 \\
2 & 17,9 & 20,9 & 12,3 & 13,9 & 68,8 & 66,2 \\
\hline
\end{tabular}

É interessante observar que nenhuma das condições otimizadas foi testada no planejamento experimental e que não houve resultados tão expressivos quanto nas duas condições otimizadas, tanto para a obtenção de pó e bixina quanto na pureza do extrato.

Nos dois casos foi observado que a relação $\mathrm{H}_{\mathrm{f}} / \mathrm{H}_{\mathrm{a}}$ foi igual, respectivamente, a 1,5 e 0,42. Assim, para se obter uma grande quantidade de pó e bixina (Caso 1), a altura da tela deve ser menor do que a altura da fonte, sendo mantida em uma posição intermediária; não tão alta de modo que as sementes não a alcancem nem tão baixa, de modo a desestabilizar o regime de jorro. Fisicamente, foi observado que esta altura intermediária do anteparo, próxima do valor do ponto central do planejamento, fazia com que as sementes atingissem a tela no final da sua trajetória de subida, aumentando o atrito sem, contudo, se chocarem fortemente contra ela. A vazão de ar e a carga de sementes devem ser altas; a primeira para aumentar a velocidade das partículas e intensificar o atrito entre elas; a segunda para prover uma quantidade suficiente de matéria-prima, objetivando obter um bom rendimento.

No Caso 2, em que a relação $\mathrm{H}_{\mathrm{f}} / \mathrm{H}_{\mathrm{a}}$ foi menor do que 1, o topo da fonte ficou longe do anteparo, ou seja, as sementes não o acertaram e acabaram anulando o seu efeito. Pode ser concluído, então, que para se obter 
um pó com elevada pureza (acima de $65 \%$ ) a altura do anteparo deve ser mais alta do que a fonte e combinada com baixas vazões de ar e cargas de semente. Um efeito similar em condições próximas às citadas foi obtido no experimento 13 (Tabela 3) que obteve como resultado um pó com aproximadamente $60 \%$ de bixina. Deve-se destacar, todavia, que nestas condições apenas uma pequena quantidade de pó será extraída, sendo interessante uma etapa subsequente de extração em outras condições, no próprio leito de jorro ou utilizando outro método, uma vez que as sementes ainda contém uma considerável quantidade residual de bixina aderida às suas paredes.

\subsection{Microscopia Eletrônica de Varredura (MEV)}

As superfícies das sementes de urucum foram analisadas utilizando a técnica de microscopia eletrônica. Três amostras de sementes foram selecionadas: sem nenhum tratamento e após extração nas 2 condições otimizadas. Aumentos de 46 e 1000 vezes foram aplicados nas imagens. As Figuras 4 $(a, b)$ mostram a superfície de uma semente intacta, sem nenhum tipo de tratamento. Nestas imagens pode ser observada uma estrutura intacta, com a presença de diversos glóbulos na superfície da semente.

Figura 4 (a,b) - Estrutura da semente intacta. Aumento de 46x (a) e 1000x (b)

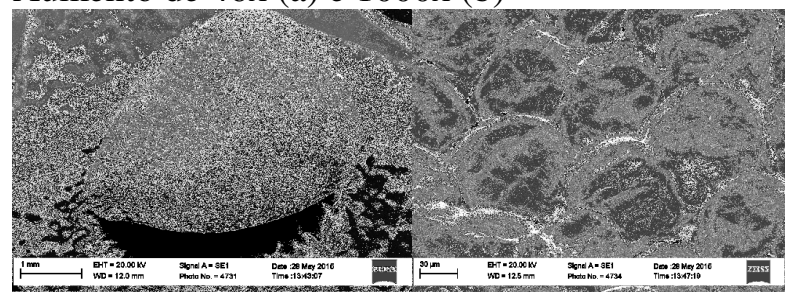

As Figuras $5(a, b)$ mostram a semente após extração nas condições otimizadas do Caso 1: Carga de sementes igual a $2500 \mathrm{~g}$, altura do anteparo igual a $40 \mathrm{~cm}$ e $Q / \mathrm{Q}_{\mathrm{jm}}$ igual a 2. A superfície das sementes apresenta-se muito mais plana do que aquela apresentada na Figura 4 (sem nenhum tipo de tratamento), devido ao quase completo esgotamento da camada de corante da superfície (Figura 5a). A Figura 5b mostra apenas uma pequena quantidade de pigmento ainda aderida à superfície.

Figura 5 (a,b) - Estrutura da semente após extração caso 1. Aumento de 46x (a) e 1000x (b)

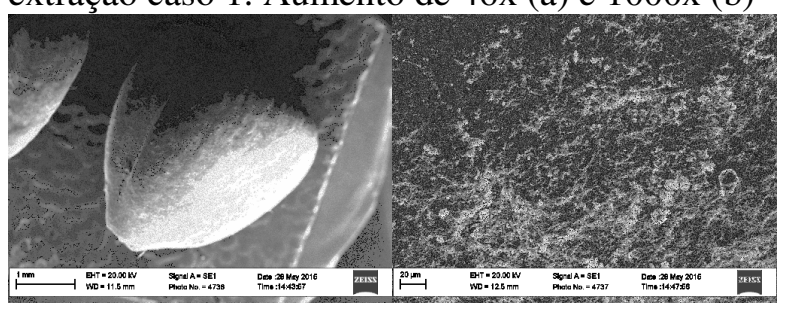

As Figuras $6(\mathrm{a}, \mathrm{b})$ mostram a semente após extração nas condições otimizadas do Caso 2: Carga de sementes igual a $1500 \mathrm{~g}$, altura do anteparo igual a $70 \mathrm{~cm}$ e $Q / Q_{j m}$ igual a 1,05. Embora a estrutura globular apresentada pelas sementes (Figura 6b) tenha permanecido, é possível observar uma superfície mais murcha, quando comparada à semente intacta (Figura 4). Este fato indica que certa quantidade de pigmento foi removida sem, contudo, extrair todo o conteúdo presente nas sementes.

Figura $6(\mathbf{a}, \mathbf{b})$ - Estrutura da semente após extração caso 2. Aumento de 46x (a) e 1000x (b)

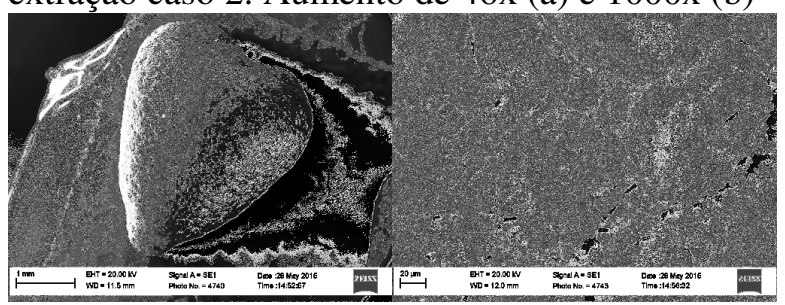

\section{CONCLUSÃO}

No presente estudo, uma unidade de leito de jorro com um anteparo de altura regulável foi utilizado com sucesso para estudar a extração mecânica da bixina das sementes de urucum. Foi estudada a influência de três variáveis (carga de 
sementes, altura de um anteparo no interior do equipamento e vazão de ar) em três respostas (massa de pó, massa de bixina e porcentagem de bixina no pó). Equações empíricas foram propostas para quantificar o efeito de cada variável, bem como de suas interações, nas respostas selecionadas. A otimização dos resultados foi feita utilizando o método algorítmico de evolução diferencial para encontrar as condições que levassem (1) - ao máximo rendimento de pó e bixina e (2) - à máxima pureza do extrato obtido. Nas condições otimizadas, os valores experimentais se aproximaram dos valores preditos em todas as situações: tanto para obtenção de pó e bixina quanto na pureza do pó obtido.

\section{NOMENCLATURA}

$\mathrm{Q}_{\mathrm{jm}}=$ vazão de jorro mínimo $\left(\mathrm{m}^{3} / \mathrm{h}\right)$

$\mathrm{M}_{\mathrm{s}}=$ massa das sementes de urucum $(\mathrm{g})$

$\mathrm{H}_{\mathrm{a}}=$ altura do anteparo metálico

$\mathrm{Q} / \mathrm{Q}_{\mathrm{jm}}=$ relação entre vazão de ar e vazão de

jorro mínimo (adimentsional)

$\mathrm{M}_{\mathrm{p}}=$ massa de pó extraído $(\mathrm{g})$

$\mathrm{M}_{\mathrm{b}}=$ massa de bixina extraída $(\mathrm{g})$

$\%_{\text {bix }}=$ porcentagem de bixina no pó $(\%)$

$\mathrm{H}_{\mathrm{f}}=$ altura máxima da fonte $(\mathrm{cm})$

$\mathrm{H}_{\mathrm{f}} / \mathrm{H}_{\mathrm{a}}=$ relação entre altura da fonte e altura do anteparo metálico (adimensional)

\section{REFERÊNCIAS}

AGNER, A.R.; BARBISAN, L.F.; SCOLASTICI, C.; SALVADORI, D.M.F. Absence of carcinogenic and anticarcinogenic effects of annatto in the rat liver medium-term assay. Food and Chem. Toxicol., v.42, p.1687-1693, 2004.

ALBUQUERQUE, C. L.; MEIRELES, M. A. de A. Defatting of annatto seeds using supercritical carbon dioxide as a pretreatment for the production of bixin: Experimental, modeling and economic evaluation of the process. The Journal of Supercritical Fluids, v. 66, p. 86-95, 2012.

ANDERSON, S. G.; NAIR, M. G.; CHANDRA, A.; MORRISON, E. Supercritical fluid carbon dioxide extraction of annatto seeds and quantification of transbixin by high pressure liquid chromatography. Phytochem Anal., v.8 p.247-249, 1997.

$\begin{array}{lcr}\text { ASSOCIATION } & \text { OF } & \text { OFFICIAL } \\ \text { ANALYTICAL } & \text { CHEMISTS } & \text { (AOAC). }\end{array}$
Official Methods of Analysis. $16^{\mathrm{a}}$ ed., Washington, 1997.

BARROZO, M. A. S.; SANTOS, K. G.; CUNHA, F. G. Mechanical Extraction of natural dye from Bixa orellanna seeds in spouted bed. Industrial Crops and Products v.45, p.279-282, 2013.

CHUYEN, H. V.; NGOC HOI, N. T. \& EUN $\mathrm{J}$-B. Improvement of bixin extraction yield and extraction quality from annatto seed by modification and combination of different extraction methods. Inter. J. Food Sci. Technol., v. 47, p. 1333-1338, 2012.

CHAO, R. R.; MULVANEY, S. J.; SANSOM, D. R.; HSIEH, F.H.; TEMPESTA, M. S. Supercritical $\mathrm{CO} 2$ extraction of annatto (Bixa orellana) pigments and some characteristics of the color extracts. J Food Sci., v.56, p.80-83, 1991.

CUNHA, F.G.; SANTOS, K.G.; ATAÍDE, C.H.; EPSTEIN, N.; BARROZO, M. A. S. Annatto Powder Production in a Spouted Bed: An Experimental and CFD Study. Industrial and Engineering Chemistry Research, v.48, p.976-982, 2009.

FOOD AND AGRICULTURE ORGANIZATION OF THE UNITED NATIONS (FAO). Combined Compendium of Food Additive Specifications, in: $67^{\text {th }}$ Joint 
FAO/WHO Expert Committee on Food Additives v.11, 2006.

MCKEOWN, G. G.; MARK, E. THE COMPOSITION OF OIL-SOLUBLE ANNATTO FOOD COLORS. Journal of the AOAC. v. 45 (3), p. 761-766, 1962.

MORAES, M. N.; ZABOT, G. L.; MEIRELES, M. A. A. Extraction of tocotrienols from annatto seeds by a pseudo continuously operated SFE process integrated with low-pressure solvent extraction for bixin production. The Journal of Supercritical Fluids, v.96, p.262-271, 2015.

NOBRE, B. P.; MENDES, R. L.; QUEIROZ, E. M.; PESSOA, F. L. P.; COELHO, J. P.; PALAVRA, A. F. Supercritical carbon dioxide extraction of pigments from Bixa orellana seeds (experiments and modeling). Braz. J. Chem. Eng., v. 23(2), p.251-258, 2006.

PASSOS, M. L.A.; MASSARANI, G.; OLIVEIRA, L. S.; FRANCA, A. S.; FREITAS, M. E. A.; BARROZO, M. A. S. Bixin Powder Production In Conical Spouted Bed Units. Drying Technology Journal, v.16(9), p.1855-1879, 1998.

PRENTICE-HERNANDEZ, C.; RUSIG, O. Extrato de urucum (Bixa orellana L.) obtido utilizando álcool etílico como solvente. Arquivos de Biologia e tecnologia, v. 35 (1), p.63-74, 1992.

PRICE, K.; STORN R. Differential evolution - a simple evolution strategy for fast optimization. Dr.Dobb's Journal, v.22, p.1824, 1997.

RODRIGUES, L. M.; ALCÁZAR-ALAY, S. C.; PETENATE, A. J.; MEIRELES, M. A. A. Bixin extraction from defatted annatto seeds.
Comptes Rendus Chimie, v.17, p.268-283, 2014.

SATYANARAYANA, A.; PRABHAKARA, P. G., RAO, D. G. Chemistry, processing and toxicology of annatto (Bixa orellana L). J Food Sci Technol., v.40(2), p.131-141, 2003.

SHUSHAMA, I. K.; AGUIAR, M. L.; OLIVEIRA, W. P.; FREITAS, L. A. P. Experimental production of annatto powders in spouted bed dryer. J Food Eng., v.59, p.9397, 2003.

SILVA, D. O.; VIEIRA, L. G. M.; LOBATO, F. S.; BARROZO, M. A. S. Optimization of the design and performance of hydrocyclones by Differential Evolution technique. Chemical Engineering and Processing: Process Intensification, v.61, p.1-7, 2012.

SILVA, G. F.; CAVALCANTI, A. S.; SOBRAL, M. C. Extraction of annatto colourants. II Proceedings of Brazilian Association of Chemistry v.43(1/2), p.58-64, 1994.

SILVA, G. F.; GAMARRA, F. M. C.; OLIVEIRA, A. L.; CABRAL F. A. Extraction of bixin from annatto seeds using supercritical carbon dioxide. Br J Chem Eng., v.25, p.419-426, 2008.

TAHAM, T.; CABRAL, F. A.; BARROZO, M. A. S. Extraction of bixin from annatto seeds using combined technologies. The Journal of Supercritical Fluids, v.100, p.175-183, 2015. 